\title{
Validation List no. 145 \\ List of new names and new combinations previously effectively, but not validly, published
}

Correspondence

Jean Euzéby

email address can be found at www.bacterio.net
The purpose of this announcement is to effect the valid publication of the following effectively published new names and new combinations under the procedure described in the Bacteriological Code (1990 Revision). Authors and other individuals wishing to have new names and/or combinations included in future lists should send three copies of the pertinent reprint or photocopies thereof, or an electronic copy of the published paper, to the IJSEM Editorial Office for confirmation that all of the other requirements for valid publication have been met. It is also a requirement of IJSEM and the ICSP that authors of new species, new subspecies and new combinations provide evidence that types are deposited in two recognized culture collections in two different countries. It should be noted that the date of valid publication of these new names and combinations is the date of publication of this list, not the date of the original publication of the names and combinations. The authors of the new names and combinations are as given below, and these authors' names will be included in the author index of the present issue. Inclusion of a name on these lists validates the publication of the name and thereby makes it available in bacteriological nomenclature. The inclusion of a name on this list is not to be construed as taxonomic acceptance of the taxon to which the name is applied. Indeed, some of these names may, in time, be shown to be synonyms, or the organisms may be transferred to another genus, thus necessitating the creation of a new combination.

\begin{tabular}{|c|c|c|c|c|}
\hline Name/author(s) & Proposed as: & Nomenclatural type ${ }^{\star}$ & Priority $\dagger$ & Reference \\
\hline Aquimarina salinaria Chen et al. 2012 & sp. nov. & Strain antisso-27 $(=$ BCRC $80080=$ LMG 25375 $)$ & 11 & 3 \\
\hline Bacillus berkeleyi Nedashkovskaya et al. $2012 \ddagger$ & sp. nov. & Strain KMM 6244 (=KCTC 12718=LMG 26357) & 8 & 12 \\
\hline Bifidobacterium biavatii Endo et al. 2012 & sp. nov. & Strain AFB23-4 (=JCM 17299=DSM 23969) & 2 & 5 \\
\hline Bifidobacterium callitrichos Endo et al. 2012 & sp. nov. & Strain AFB22-5 (=JCM 17296=DSM 23973) & 2 & 5 \\
\hline Bifidobacterium reuteri Endo et al. 2012 & sp. nov. & Strain AFB22-1 (=JCM 17295=DSM 23975) & 2 & 5 \\
\hline Bifidobacterium saguini Endo et al. 2012 & sp. nov. & Strain AFB23-1 (=JCM 17297=DSM 23967) & 2 & 5 \\
\hline Bifidobacterium stellenboschense Endo et al. 2012 & sp. nov. & Strain AFB23-3 (=JCM 17298=DSM 23968) & 2 & 5 \\
\hline Chromatocurvus corrig. Csotonyi et al. $2012 \$$ & gen. nov. & $\begin{array}{l}\text { Chromatocurvus halotolerans corrig. } \\
\text { Csotonyi et al. } 2012\end{array}$ & 1 & 4 \\
\hline Chromatocurvus halotolerans corrig. Csotonyi et al. $2012 \$$ & $\$$ sp. nov. & Strain EG19 (=DSM 23344=VKM B-2659) & 1 & 4 \\
\hline Desulfosporosinus acidiphilus Alazard et al. 2012 & sp. nov. & Strain SJ4 (=DSM 22704=JCM 16185) & 15 & 1 \\
\hline Flavobacteriia Bernardet 2012 & class. nov. & Flavobacteriales Bernardet 2012 & 4 & 2 \\
\hline Flavobacterium oncorhynchi Zamora et al. 2012 & sp. nov. & Strain 631-08 $(=$ CECT 7678=CCUG 59446 $)$ & 3 & 17 \\
\hline Halogranum salarium Kim et al. 2012 & sp. nov. & Strain B-1 $(=$ KCTC $4066=$ DSM 23171) & 16 & 8 \\
\hline Leucobacter denitrificans Weon et al. 2012 & sp. nov. & Strain M1T8B10 (=KACC $14055=$ NBRC 106309) & 13 & 15 \\
\hline Maricurvus Iwaki et al. 2012 & gen. nov. & Maricurvus nonylphenolicus Iwaki et al. 2012 & 7 & 7 \\
\hline Maricurvus nonylphenolicus Iwaki et al. 2012 & sp. nov. & Strain KU41E (=JCM 17778=KCTC 23758)॥ & 7 & 7 \\
\hline Methylobacterium bullatum Hoppe et al. 20129 & sp. nov. & Strain F3.2 (=DSM 21893=LMG 24788) & 12 & 6 \\
\hline Methylobacterium longum Knief et al. 2012 & sp. nov. & Strain $440(=$ DSM $23933=$ CECT 7806$)$ & 6 & 9 \\
\hline Natranaerovirga Sorokin et al. 2012\# & gen. nov. & Natranaerovirga pectinivora Sorokin et al. 2012 & 14 & 14 \\
\hline Natranaerovirga hydrolytica Sorokin et al. 2012 & sp. nov. & Strain APP2 (=DSM 24176=UNIQEM U806) & 14 & 14 \\
\hline Natranaerovirga pectinivora Sorokin et al. 2012 & sp. nov. & Strain AP3 (=DSM 24629=UNIQEM U805) & 14 & 14 \\
\hline Nonlabens agnitus Yi and Chun 2012 & sp. nov. & Strain JC2678 (=KACC $14155=\mathrm{JCM} 17109)$ & 17 & 16 \\
\hline Poseidonocella Romanenko et al. 2012 & gen. nov. & Poseidonocella pacifica Romanenko et al. 2012 & 5 & 13 \\
\hline
\end{tabular}


cont.

\begin{tabular}{|c|c|c|c|c|}
\hline Name/author(s) & Proposed as: & Nomenclatural type* & Priority $\dagger$ & Reference \\
\hline Poseidonocella pacifica Romanenko et al. 2012 & sp. nov. & $\begin{array}{l}\text { Strain KMM } 9010(=\text { NRIC } 0794= \\
\text { JCM } 17310=\text { KCTC 23693 })^{* *}\end{array}$ & 5 & 13 \\
\hline Poseidonocella sedimentorum Romanenko et al. 2012 & sp. nov. & $\begin{array}{c}\text { Strain KMM } 9023(=\text { NRIC } 0796= \\
\text { JCM } 17311=\text { KCTC 23692) } \dagger \dagger\end{array}$ & 5 & 13 \\
\hline Pseudomonas benzenivorans Lang et al. 2012 & sp. nov. & Strain DSM 8628 (=CIP 109857) & 10 & 10 \\
\hline Pseudomonas saponiphila Lang et al. 2012 & sp. nov. & Strain DSM 9751 (=CIP 109856) & 10 & 10 \\
\hline Thermovorax Mäkinen et al. 2012 & gen. nov. & Thermovorax subterraneus Mäkinen et al. 2012 & 9 & 11 \\
\hline Thermovorax subterraneus Mäkinen et al. 2012 & sp. nov. & Strain 70B (=DSM 21563=JCM 15541) & 9 & 11 \\
\hline
\end{tabular}

For references to Validation Lists 1-71, see Int J Syst Bacteriol 49 (1999) 1325. Lists 72-144 were published in Int J Syst Evol Microbiol 50 (2000) 3, 423, 949, 1415, 1699, 1953; and 51 (2001) 1, 263, 793, 1229, 1619, 1945; and 52 (2002) 3, 685, 1075, 1437, 1915; and 53 (2003) 1, 373, 627, 935, 1219, 1701; and 54 (2004) 1, 307, 631, 1005, 1425, 1909; and 55 (2005) 1, 547, 983, 1395, 1743, 2235; and 56 (2006) 1, 499, 925, 1459, 2025, 2507; and 57 (2007) 1, 433, 893, 1371, 1933, 2449; and 58 (2008) 1, 529, 1057, 1511, 1993, 2471; and 59 (2009) 1, 451, 923, 1555, 2129, 2647; and 60 (2010) 1, 469, 1009, 1477, 1985, 2509; and 61 (2011) 1, 475, 1011, 1499, 2025, 2563; and 62 (2012) 1, 473.

${ }^{*}$ Abbreviations of culture collections cited in this list can be found at http://ijs.sgmjournals.org/site/misc/collections.xhtml $\dagger$ Priority number assigned according to the date the documentation and request for validation are received.

$\ddagger$ The correct etymology of the specific epithet should be the following: ber.ke'ley.i. N.L. gen. masc. n. berkeleyi of Berkeley, named after Roger C. W. Berkeley (1937-2010), the famous English microbiologist who greatly contributed to Bacillus taxonomy.

\$The original spelling of the generic name, Chromocurvus (sic), has been corrected on validation according to Rule 61. The etymology is the following: chro.ma.to.cur'vus. Gr. n. chroma, -atos colour; L. masc. adj. curvus curved, bent; N.L. masc. n. chromatocurvus the coloured curved micro-organism.

IIThe accession number KCTC 23758 has been provided at the time of request for valid publication of the name.

SBased on the strain history of the type strain of Methylobacterium bullatum, it appears that strain F3.2 has also been used as the type strain of 'Methylobacterium funariae' [see: Schauer, S. \& Kutschera, U. (2011). A novel growth-promoting microbe, Methylobacterium funariae sp. nov., isolated from the leaf surface of a common moss. Plant Signal Behav 6, 510-515.]

\#The correct etymology of the generic name should be the following: Natr.a.na.e.ro.vir'ga. N.L. n. natron derived from Arabic natrun, soda (sodium carbonate); Gr. pref. an not; Gr. n. aer, aeros air; L. fem. n. virga rod; N.L. fem. n. Natranaerovirga an anaerobic rod living in soda.

${ }^{*}$ The accession number KCTC 23693 has been provided at the time of request for valid publication of the name.

$\dagger \dagger$ The accession number KCTC 23692 has been provided at the time of request for valid publication of the name.

\section{References}

1. Alazard, D., Joseph, M., Battaglia-Brunet, F., Cayol, J.-L. \& Ollivier, B. (2010). Desulfosporosinus acidiphilus sp. nov.: a moderately acidophilic sulfate-reducing bacterium isolated from acid mining drainage sediments New taxa: Firmicutes (Class Clostridia, Order Clostridiales, Family Peptococcaceae). Extremophiles 14, 305-312.

2. Bernardet, J.-F. (2011). Class II. Flavobacteriia class. nov. In Bergey's Manual of Systematic Bacteriology, 2nd edn, vol. 4, p. 105. Edited by N. R. Krieg, J. T. Staley, D. R. Brown, B. P. Hedlund, B. J. Paster, N. L. Ward, W. Ludwig \& W. B. Whitman. New York: Springer.

3. Chen, W.-M., Sheu, F.-S. \& Sheu, S.-Y. (2012). Aquimarina salinaria sp. nov., a novel algicidal bacterium isolated from a saltpan. Arch Microbiol 194, 103-112.

4. Csotonyi, J. T., Stackebrandt, E., Swiderski, J., Schumann, P. \& Yurkov, V. (2011). Chromocurvus halotolerans gen. nov., sp. nov., a gammaproteobacterial obligately aerobic anoxygenic phototroph, isolated from a Canadian hypersaline spring. Arch Microbiol 193, 573-582.

5. Endo, A., Futagawa-Endo, Y., Schumann, P., Pukall, R. \& Dicks, L. M. T. (2012). Bifidobacterium reuteri sp. nov., Bifidobacterium callitrichos sp. nov., Bifidobacterium saguini sp. nov., Bifidobacterium stellenboschense sp. nov. and Bifidobacterium biavatii sp. nov. isolated from faeces of common marmoset (Callithrix jacchus) and red-handed tamarin (Saguinus midas). Syst Appl Microbiol 35, 92-97.

6. Hoppe, T., Peters, K. \& Schmidt, F. (2011). Methylobacterium bullatum sp. nov., a methylotrophic bacterium isolated from Funaria hygrometrica. Syst Appl Microbiol 34, 482-486.

7. Iwaki, H., Takada, K. \& Hasegawa, Y. (2012). Maricurvus nonylphenolicus gen. nov., sp. nov., a nonylphenol-degrading bacterium isolated from seawater. FEMS Microbiol Lett 327, 142-147.

8. Kim, K. K., Lee, K. C. \& Lee, J.-S. (2011). Halogranum salarium sp. nov., a halophilic archaeon isolated from sea salt. Syst Appl Microbiol 34, 576-580.

9. Knief, C., Dengler, V., Bodelier, P. L. E. \& Vorholt, J. A. (2012). Characterization of Methylobacterium strains isolated from the phyllosphere and description of Methylobacterium longum sp. nov. Antonie van Leeuwenhoek 101, 169-183.

10. Lang, E., Burghartz, M., Spring, S., Swiderski, J. \& Spröer, C. (2010). Pseudomonas benzenivorans sp. nov. and Pseudomonas saponiphila sp. nov., represented by xenobiotics degrading type strains. Curr Microbiol 60, 85-91.

11. Mäkinen, A. E., Kaksonen, A. H. \& Puhakka, J. A. (2009). Thermovorax subterraneus, gen. nov., sp. nov., a thermophilic hydrogen-producing bacterium isolated from geothermally active underground mine. Extremophiles 13, 505-510. 
12. Nedashkovskaya, O. I., Van Trappen, S., Frolova, G. M. \& De Vos, P. (2012). Bacillus berkeleyi sp. nov., isolated from the sea urchin Strongylocentrotus intermedius. Arch Microbiol 194, 215-221.

13. Romanenko, L. A., Tanaka, N., Svetashev, V. I. \& Kalinovskaya, N. I. (2012). Poseidonocella pacifica gen. nov., sp. nov. and Poseidonocella sedimentorum sp. nov., novel alphaproteobacteria from the shallow sandy sediments of the Sea of Japan. Arch Microbiol 194, 113-121.

14. Sorokin, D. Y., Tourova, T. P., Panteleeva, A. N., Kaparullina, E. N. \& Muyzer, G. (2012). Anaerobic utilization of pectinous substrates at extremely haloalkaline conditions by Natranaerovirga pectinivora gen. nov., sp. nov., and Natranaerovirga hydrolytica sp. nov., isolated from hypersaline soda lakes. Extremophiles 16, 307-315.
15. Weon, H.-Y., Anandham, R., Tamura, T., Hamada, M., Kim, S.-J., Kim, Y.-S., Suzuki, K. \& Kwon, S.-W. (2012). Leucobacter denitrificans sp. nov., isolated from cow dung. J Microbiol 50, 161-165.

16. Yi, H. \& Chun, J. (2012). Unification of the genera Nonlabens, Persicivirga, Sandarakinotalea and Stenothermobacter into a single emended genus, Nonlabens, and description of Nonlabens agnitus sp. nov. Syst Appl Microbiol 35, 150-155.

17. Zamora, L., Fernández-Garayzábal, J. F., Svensson-Stadler, L. A., Palacios, M. A., Dominguez, L., Moore, E. R. B. \& Vela, A. I. (2012). Flavobacterium oncorhynchi sp. nov., a new species isolated from rainbow trout (Oncorhynchus mykiss). Syst Appl Microbiol 35, 86-91. 